\title{
Myeloid Sarcoma in a One-Month-Old Infant
}

\author{
Chihiro Wakusawa Taku Fujimura Akira Hashimoto \\ Setsuya Aiba
}

Department of Dermatology, Tohoku University Graduate School of Medicine, Sendai, Japan

\section{Key Words}

Myeloid sarcoma $\cdot$ CD4 $\cdot$ Hematopoietic neoplasm

\begin{abstract}
Myeloid sarcoma, which sometimes accompanies recurrent systemic leukemia, is a rare manifestation of congenital leukemia. We describe the case of a 1-month-old Japanese girl with myeloid sarcoma, who did not fulfill the criteria of systemic leukemia. The physical examination revealed a bruise-like plaque on the right side of her chest. Histopathologically, there were CD4+ CD56- CD68+ atypical large lymphocytes densely infiltrating the upper dermis and the subcutaneous tissue. Biopsy specimens from bone marrow contained only $3.4 \%$ of blast cells, showing an abnormality of the mixed-lineage leukemia gene. The skin lesions disappeared 10 days after chemotherapy, and there was no recurrence for one and a half years.
\end{abstract}

\section{Introduction}

Myeloid sarcoma (MS), or granulocytic sarcoma, is a lesion characterized by the presence of one or more tumor masses consisting of myeloblasts or immature myeloid cells at sites other than the bone marrow, with the skin being the site of predilection.

The recent World Health Organization (WHO) classification has proposed the subdivision of MS into several histotypes, but the exact incidence of this disease has not been established yet [1]. Because MS expresses various differentiation markers and is subdivided immunohistochemically into several categories, immunophenotyping is essential to avoid misdiagnosis and inappropriate treatment [2, 3]. In addition, MS is associated with a variety of chromosomal abnormalities as well as mixed-lineage leukemia (MLL) rearrangements.

In this report, we describe a case of MS with multifocal bruise-like plaques over the trunk and extremities that showed abnormalities of the MLL gene and chromosomes. 


\section{Case Report}

A 1-month-old Japanese girl presented to our outpatient clinic with an asymptomatic plaque on her chest. At her initial visit, physical examination revealed a $13 \times 16 \mathrm{~mm}$, red-violaceous, bruise-like plaque on the right side of her chest (fig. 1). One week after her arrival, disseminated bruise-like purpura was detected on her extremities. A biopsy specimen from the chest revealed atypical cells

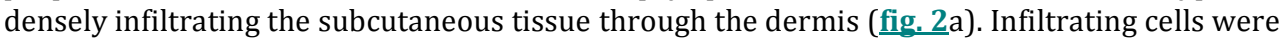
medium-sized, with a high nuclear/cytoplasmic ratio and prominent nucleoli (fig. 2 b).

Immunohistochemical staining revealed that these infiltrating cells mainly consisted of CD4+ cells (fig. 2c), were diffusely CD68 positive (fig. 2d) and CD56 negative. Infiltrating cells were also negative for CD3, CD5, CD8, CD10, CD20, CD34, CD45, CD79 $\alpha$, neutrophil elastase, and myeloperoxidase. The Ki67 score was approximately $80 \%$. Biopsy specimens from bone marrow contained only $3.4 \%$ blast cells; however, biopsies of the skin showed abnormalities in the MLL gene. Moreover, the division inside the MLL gene area (11q23) was detected in about $84 \%$ of counted cells by fluorescence in situ hybridization. Based on the above data, we diagnosed this patient as having MS with monoblastic/monocytic differentiation [1]. The patient was treated with the so-called MLL99 regimen consisting of etoposide, cytarabine, mitoxantrone, idarubicin, methotrexate and hydrocortisone. The skin lesions disappeared 10 days after the administration of MLL99, and there was no evidence of recurrence for one and a half years.

\section{Discussion}

MS usually presents as a single mass, although cases of MS with multiple skin lesions have also been reported [3]. In this report, we present a case of MS with multifocal bruise-like plaques over the trunk and extremities.

MS is a rare condition characterized by the occurrence of one or more tumor masses consisting of immature myeloid cells presenting at an extramedullary site. It may develop de novo or concurrently with acute myeloid leukemia (AML), myeloproliferative disorder (MPD) or myelodysplastic syndrome (MDS) [3, 4]. Indeed, Pileri et al. [3] reviewed 92 cases of MS and suggested that 25\% of the patients developed de novo disease, and the other cases had AML, MPD or MDS simultaneously (32\%) or previously (35\%).

As for the prognosis of this disorder, the presence of MPD, MDS or AML is reported to be a negative prognostic factor [3,5]. By contrast, de novo MS has been described as sensitive to radiotherapy and/or chemotherapy, with possible prolonged survival. However, recent studies suggest that either highly specific drugs or aggressive regimens should also be applied to de novo MS, if the aim is to achieve stable remission and cure. In our present case, we administered the MLL99 regimen and achieved complete remission for one and a half years. Though there is no definitive consensus concerning the optimal treatment for MS, our present case suggests that intensive chemotherapy is necessary to induce complete remission of MS. 


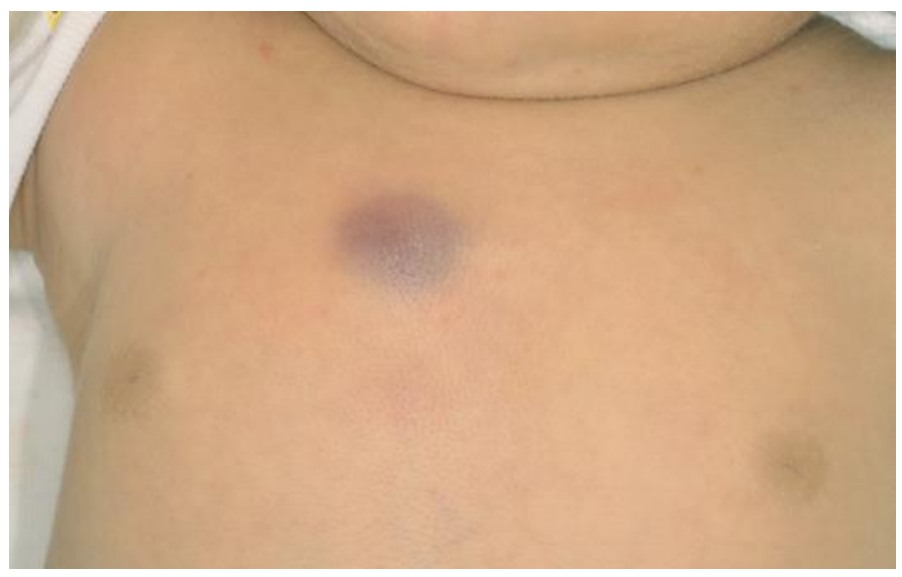

Fig. 1. Red-violaceous bruise-like plaque, $13 \times 16 \mathrm{~mm}$ in size, on the right side of the chest.
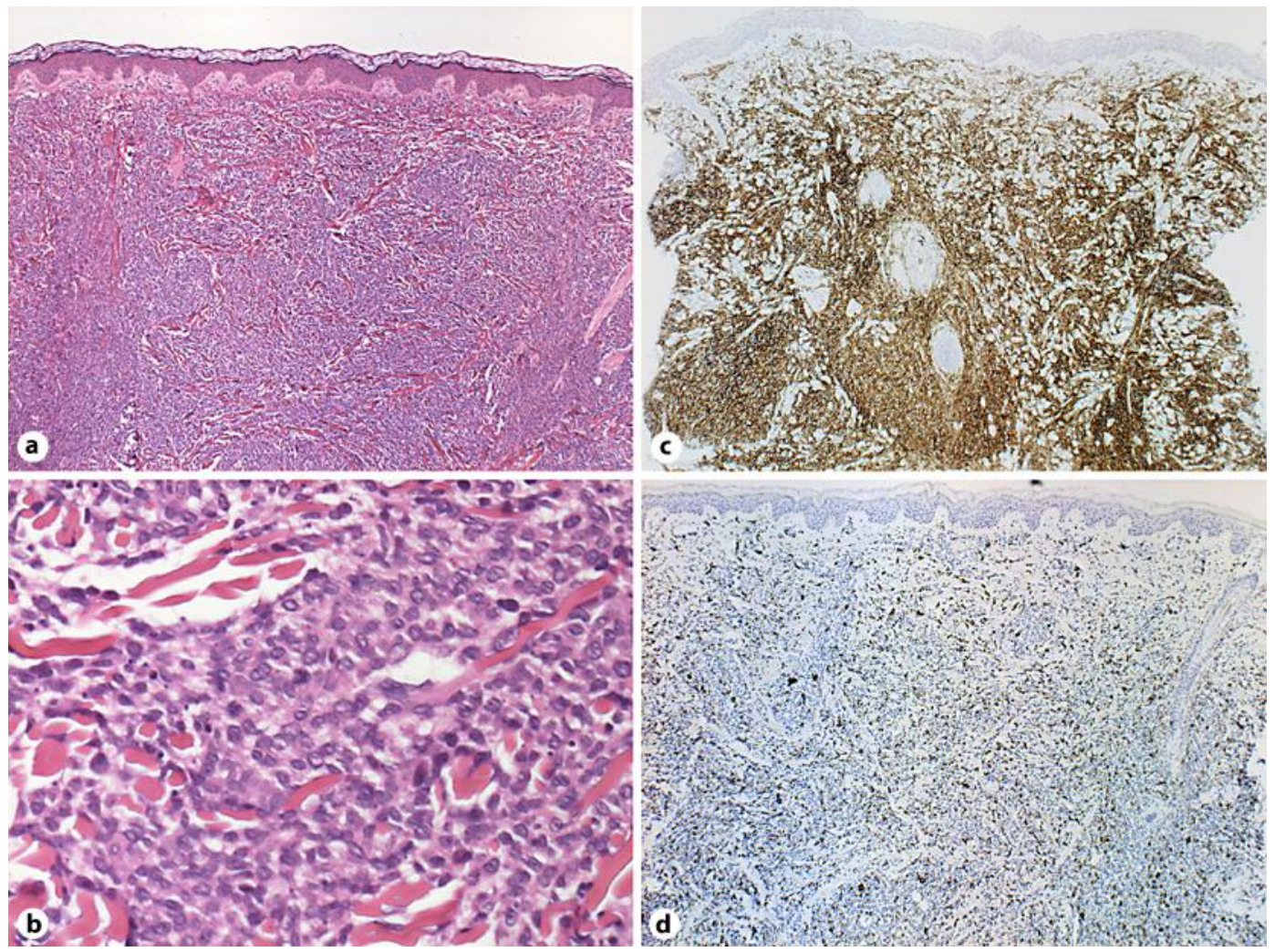

Fig. 2. Atypical cells densely infiltrating the dermis and the subcutaneous tissue (a). Infiltrating cells were medium-sized, with a high nuclear/cytoplasmic ratio and prominent nucleoli (b). Immunohistochemical staining for CD4 (c) and CD68 (d). Original manifestation $\times 50(a, c, d)$ and $\times 400($ b) . 


\section{References}

1 Swerdlow SH, Campo E, Harris NL, Jaffe ES, Pileri SA, Stein H, Thiele J, Vardiman JW: WHO Classification of Tumours of Haematopoietic and Lymphoid Tissues, ed 4. Lyon, IARC, 2008.

-2 Nakagawa S, Tagami H, Ichinohasama R, Aiba S: Rapid growing cobblestone-like nodules as a manifestation of myeloid sarcoma. Acta Derm Venereol 2008;88:633-634.

3 Pileri SA, Ascani S, Cox MC, Campidelli C, Bacci F, et al: Myeloid sarcoma: clinico-pathologic, phenotypic and cytogenetic analysis of 92 adults patients. Leukemia 2007;21:340-350.

4 Campidelli C, Agostinelli C, Stitson R, Pileri SA: Myeloid sarcoma: extramedullary manifestation of myeloid disorders. Am J Clin Pathol 2009;132:426-437.

5 Magro CM, Porcu P, Schaefer J, Erter JW, Furman RR, Shitabata PK, Crowson AN: Cutaneous CD4+ CD56+ hematologic malignancies. J Am Acad Dermatol 2010;63:292-308. 